\title{
Parametric Analysis of Mobile Ad-Hoc Network Environment
}

\author{
M.Shahaya Sheela ${ }^{1}$, A.Sivanantha Raja ${ }^{2}$ and V.R.Sarma Dhulipala ${ }^{3}$ \\ ${ }^{1}$ Assistant Professor, Dept of ECE, Centhuran College of Engineering, Tiruchirappalli, Tamil Nadu, India \\ ${ }^{2}$ Associate Professor, Dept of ECE, AC.Tech, Karaikudi, Tamil Nadu, India \\ ${ }^{3}$ Assistant Professor, Dept of Physics, Anna University of Technology, Tiruchirappalli, Tamil Nadu, India
}

\begin{abstract}
A mobile Ad-Hoc Network (MANET) is a collection of wireless nodes that communicate with each other without any infrastructure. Present day mobile ad hoc networks grow in size and the associated complexity warrant the need to explore the suitability of the various simulation parameters during their execution. This paper presents a study on the timing Analysis for common MANET (mobile ad hoc network) parameters.
\end{abstract}

Index terms: AODV, DSR, MANET, Random waypoint mobility model, Mobile Node, Transmission Range and scalability

\section{INTRODUCTION}

Mobile ad hoc networks (MANETs) is a self-configuring network of mobile routers (and associated hosts) connected by wireless links. Communication must be set up and maintained on the fly over mostly by wireless links. Each node of a network can both route and forward data [1]. The exploding demand for computing and communication on the move has led to reliance for ad hoc networks. Although substantial attempts have been made on research towards design and development of ad hoc network parameters, there is relatively little understanding of their behaviour in terms of the performance by comparing execution times as the system is scaled up [2] \& [8].

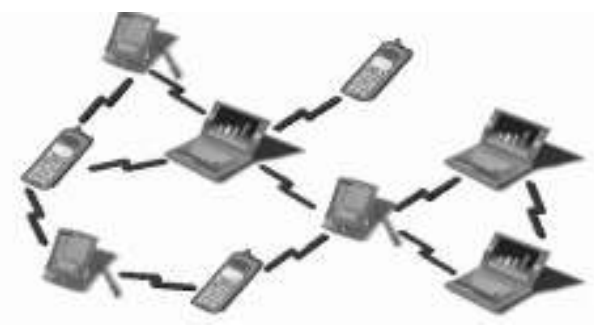

Figure 1

First, it is unclear whether any existing ad hoc application traffics can be used to successfully perform within the given terrain dimensions which will be necessary in large scale wireless networks. Second, it is also ambiguous that which of the preceding traffics is likely to perform better as the size of the network grows [3]. In this paper represents the execution time analysis by varying different MANET simulation parameters. Because execution time is a main parameter for analyzing the performance of mobile ad-hoc network. Here we are using the simulation tool GloMoSim for large scale scenarios to obtain execution time analysis by varying different simulation parameters. Because it is giving more accuracy while constructing large scale scenarios.

\section{EXECUTION TIME}

In Mobile ad hoc network (MANET) consist of mobile hosts without any infrastructure. Here the Execution time is the essential parameter in performance analysis for the research peoples. Execution time is the time for executing a particular scenario.[2]. Here in GloMosim execution time is measured by

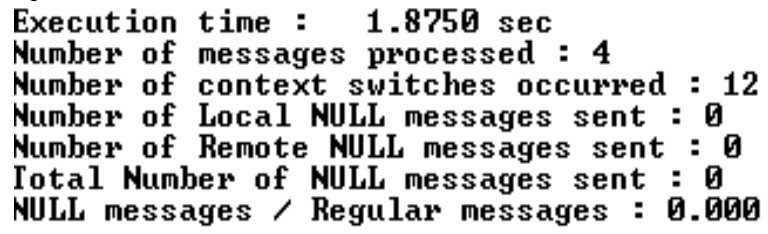

\section{DSR}

Dynamic Source Routing adopts a similar on-demand approach as AODV regarding the route discovery and maintenance processes. A key difference of DSR from AODV and other on-demand protocols is the use of source routing, where the source node specifies the complete sequence of intermediate nodes for each data packet to reach its destination. The source route information is carried by the header of the data packet. The advantage of source routing is that no additional mechanism is needed to detect routing loops. [1] \& [9].

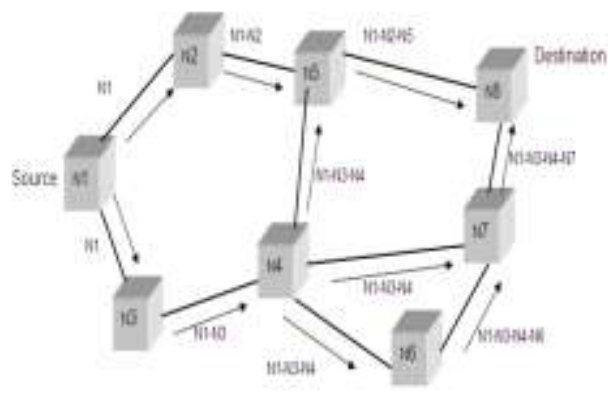

Figure 2. route discovery of DSR

The data structures DSR uses to store routing information is route cache, with each cache entry storing one specific route from the source to a destination.

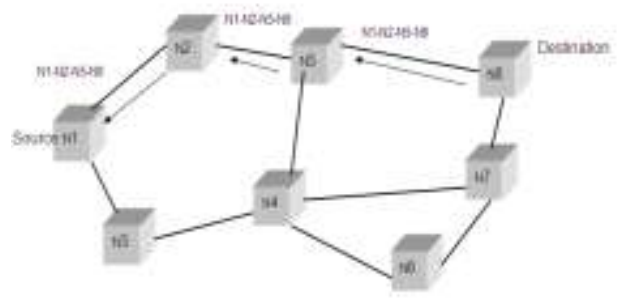

Figure 3. route maintenance of DSR 
DSR makes very aggressive use of the source routing information. Every intermediate node caches the source route carried in a data packet it forwards, and the following optimization rules to DSR have also been proposed:

3.1. Salvaging: If an intermediate node discovers that the next hop in the source route is unreachable; it can replace the source route in the data packets with a route in the data packets with a route from its own cache.

3.2. Gratutious Route Repair: A source node notified error of the packets it originates propagates the error notification to its neighbors by biggy-backing it on its next route request. This helps clean up the caches of other nodes in the network that may have the failed link in one of the cached source routes.

3.3. Promiscous Listening: when a node overhears a packet that is addressed to another node, it adds the source route information into its own route caches. The node also checks if the packet could be routed via itself to gain a shorter route.[6]

\section{AODV}

A node running AODV initiates a route discovery process only when it has data packets to send and it does not know any route to the destination node that is route discovery AODV is on-demand.

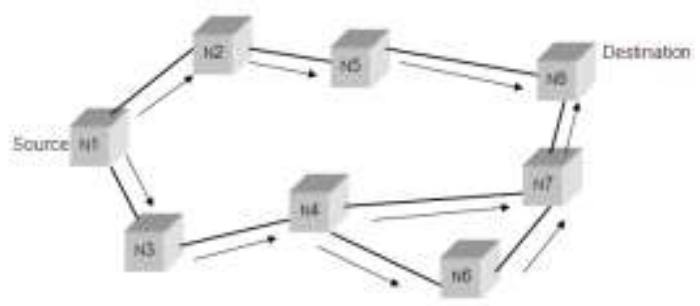

Figure 4. Route discovery of AODV

During a route discovery process, the source node broadcasts a route query packet to its neighbors. If any of the neighbors has a route to the destination it replies to the query with a route reply packet; otherwise, the neighbors rebroadcast the route query packet. Finally some query packets reach the destination or nodes that know a route to the destination. At that time, a reply packet is produced and transmitted tracing back the route traversed by the query packet. To handle the case in which a route does not exist, or the query or reply packets are lost, the source node rebroadcasts the query packet if no reply is received by the source after a time-out.

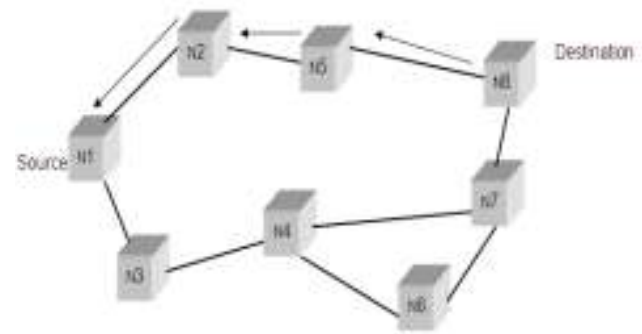

Figure.5. route maintenance of AODV

A path maintenance process is used by AODV to monitor the operation of a route being used. If a source node receives the notification of broken link, it can re-initiate the route discovery processes to find a new route to the destination. If a destination or an intermediate node detects a broken link, it sends special messages to the affected source node.

AODV uses a routing table to specify distances to destinations. It uses sequence numbers maintained at each destination to determine the freshness of routing information and to prevent routing loops.

The recent specification AODV suggests and optimization to AODV; it uses and expanding ring search to discover routes to an unknown destination. In the expanding ring search increasingly larger neighborhoods are searched to find the destination. [6]

\section{RANDOM WAYPOINT MODEL}

In this paper we used random waypoint mobility model for analyzing execution time with MANET routing protocols.

Random Waypoint (RWP) model is a commonly used synthetic model for mobility, e.g., in Ad Hoc networks. It is an elementary model which describes the movement pattern of independent nodes by simple terms. [11].

Briefly, in the RWP model:

- Each node moves along a zigzag line from one waypoint to the next

- The waypoints are uniformly distributed over the given convex area, e.g. unit disk.

- At the start of each leg a random velocity is drawn from the velocity distribution (in the basic case the velocity is constant 1)

- Optionally, the nodes may have so-called "thinking times" when they reach each waypoint before continuing on the next leg, where durations are independent and identically distributed random variables

\section{GloMosim}

GloMoSim (Global Mobile Information System Simulator) is a library based sequential and parallel simulator for wireless networks. It is designed as a set of library modules, each of which simulates a specific wireless communication protocol in the protocol stack. The library has been developed using PARSEC a C- based parallel simulation language. New protocols and modules can be programmed and added to the library using this language. GloMoSim has been designed to be extensible and composable. It has been implemented on both shared memory and distributed memory computers and can be executed using a variety of synchronization protocols. GloMoSim runs with the help of PARSEC. [5]

\subsection{PARSEC}

PARSEC (Parallel Simulation Environment for Complex system) is a C-based simulation language developed by the Parallel Computing Laboratory at UCLA, for sequential and parallel execution of discrete-event simulation models. It can also be used as a parallel programming language. PARSEC runs on several platforms, including most recent UNIX variants as well as window. $[5,7]$.

\subsection{GloMoSim Network Architecture}

The networking stack is decomposed into a number of layers as shown Fig.6.2. A number of protocols have been developed at each layer and models of these protocols or layers can be developed at different levels of granularity [5]. 


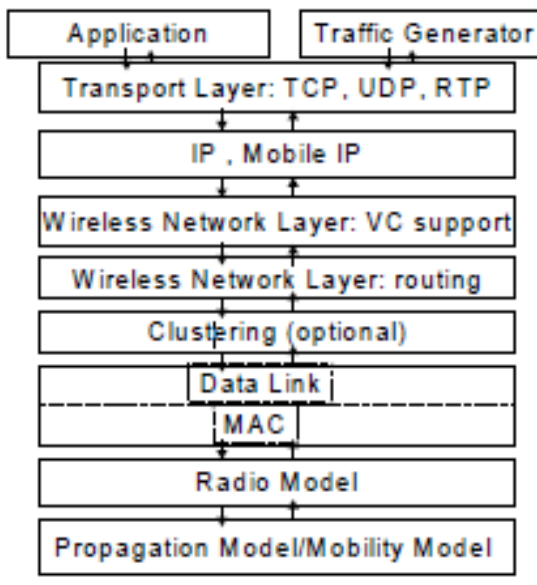

Fig.6 GloMoSim Network Architecture

\section{DESIGN OF THE EXPERIMENT \& SIMULATION SETUP}

The method for analyzing the execution time is to begin with a carefully Designed base configuration and network scenario for the experiment, and to vary The node density and mobility at a time to stress the network in different directions. Careful selection of these control parameters enables us to assess and isolate the Effect of network size, with fixed application traffic CBR. In addition, design of the base condition, network topology, and routing are to be taken into account the Real networks for which the results should be applicable.

\subsection{Mobility model}

Nodes in the simulation set up move according to a model that is well known as the "random waypoint" model. The movement scenario files we used for each Simulations are characterized by a pause time. Each node begins the simulation by remaining stationary for pause time seconds. It then selects a random destination in the $1500 \mathrm{~m} \times 1500 \mathrm{~m}$ space and moves to that destination at a speed distributed Uniformly between $0 \mathrm{mps}$ and a maximum speed of $10 \mathrm{mps}$. Upon reaching the Destination, the node pauses again for pause time seconds, selects another Destination, and proceeds there as previously described, repeating this behavior for the duration of the simulation. Each simulation ran for 200 seconds of Simulated time. We ran our simulations with movement patterns generated for a Fixed pause time of 30 Seconds.

\subsection{Application Traffic}

As the goal of our simulation was to compare the performance of each routing Protocol, we chose our application traffic sources to be constant bit rate (CBR) Sources [2] .When defining the parameters of the communication model, we Experimented with sending rates of 10 packets per second and packet sizes of 512 Bytes to observe the consistency.

\subsection{Experiment Modeling:}

- Traffic load is modeled using constant bit rate(CBR) data stream

- Movement of nodes is modeled using the "random waypoint model"

- Terrain dimension is not fixed, initial is 500,500

- Initial transmission range is 15

- No of nodes used in the simulation 10, 70, 125, 200
- Bandwidth of each link is $2 \mathrm{Mbps}$

- The simulation time is 60 seconds for all cases TCP is NOT used in transport layer as it performs flow control and retransmission The simulator used was Glomosim

\subsection{AODV, DSR Vs Execution Time:}

Here we compared the execution time analysis by using AODV, DSR routing protocols. And we noticed the Execution time varies as follows

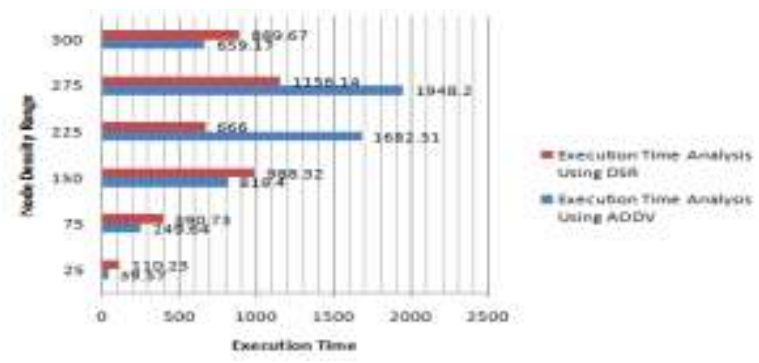

\subsection{Mobility Vs Execution time}

Here we analysed the Execution time by using with and without mobilty (RWP Model)

As shown in the following figure.the result shows the execution time varies high when using mobility.

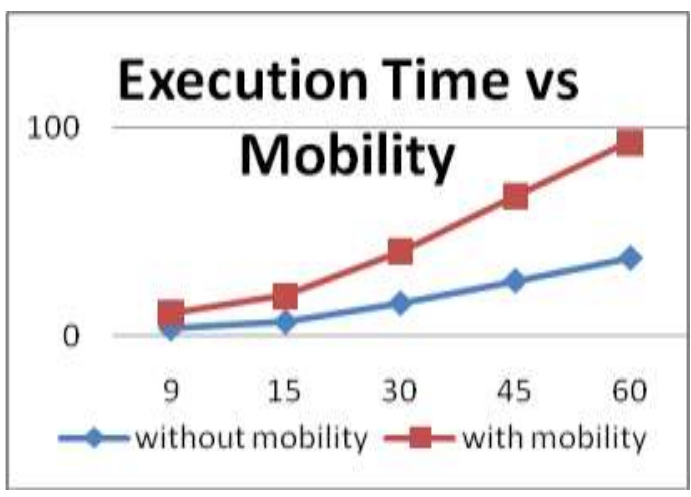

\subsection{Node density Vs Execution Time}

Here we compared the execution time by varying no of mobile nodes. As the result the Execution time increases when increasing the node density.

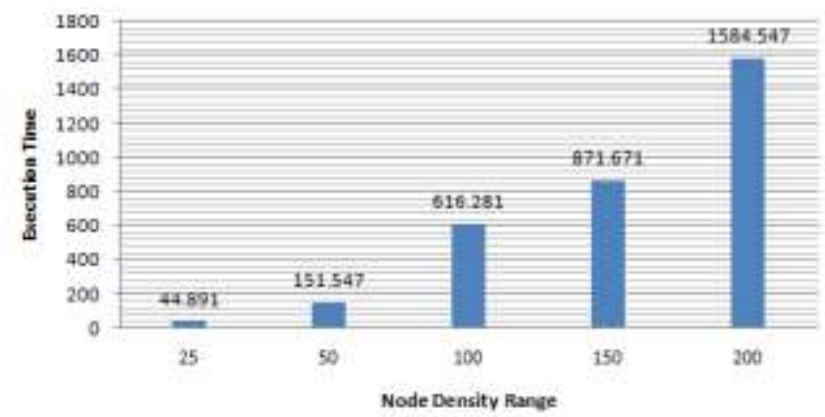




\subsection{Processor Vs Execution Time:}

Here we compared the execution time by using two different type of processors to the same scenario.As the result shows the Execution time is high for advanced processors while compared with low speed processors.

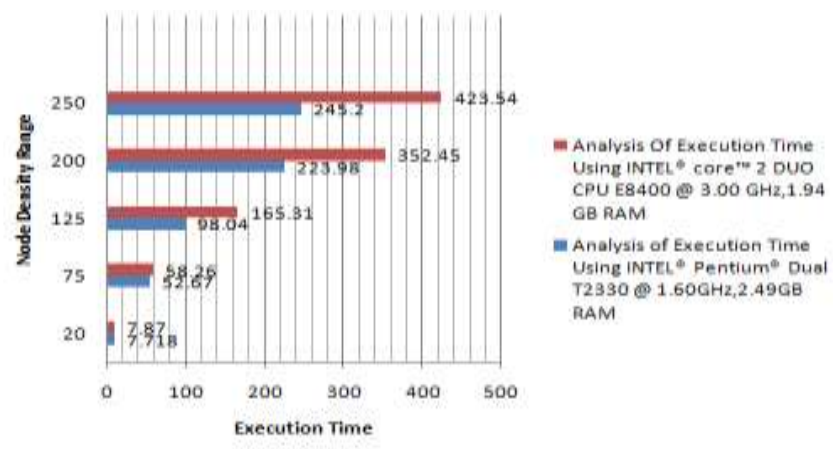

\subsection{Terrain Range Vs Execution Time:}

In the same scenario we changed the terrain dimensions, the execution time varies or high for increasing the terrain dimension.the result is following in the figure

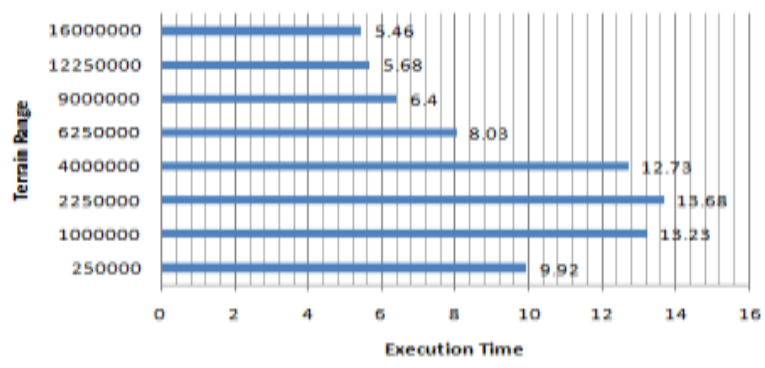

\subsection{Transmission Range Vs Execution}

\section{Time:}

In Mobile Ad hoc Network the mobile nodes are battery constraint.so the transmission range is very essential parameter to analyse the Execution time. The following graph shown the analysis of execution time with varying transmission range.

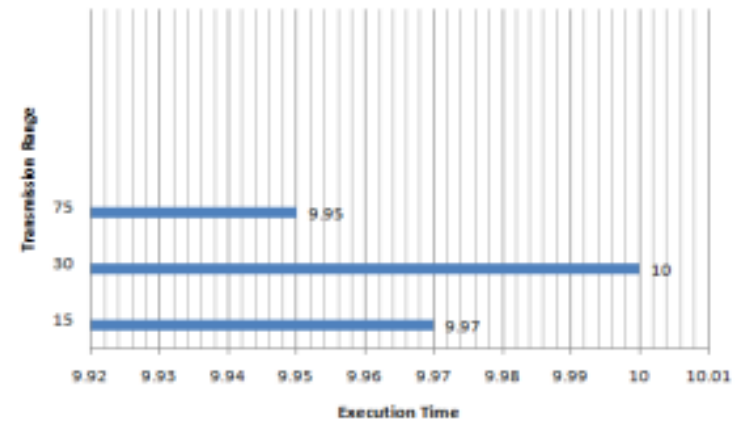

\section{CONCLUSION}

The work was initiated with an intention of carrying out exhaustive study of the performance issues of large scale MANETs. We obtained convincing results for the execution times and it's affected while changing network parameters vs. real times in large scale scenario.

\section{FUTURE WORK}

The work was initiated with an intention of carrying out exhaustive study of the performance issues of large scale MANETs. We obtained convincing results for the execution times and deviations of execution Vs real times for a selected scenario when scaled up. As reported in MOBIHOC Survey [10], we can say that a maximum of 7interactive runs produced consistent results for our experiment.

\section{REFRENCES}

[1]. Tope. M.A, McEachen, J.C, and Kinney. A.C, "Ad-hoc network routing using co-operative diversity", Advanced Information Networking and Applications, IEEE Conference, 18-20 April 2006.

[2]. V.R. Sarma Dhulipala, RM.Chandrasekaran, R.Prabakaran " Timing Analysis and Repeatability Issues of Mobile Ad-Hoc Networking Application traffics in Large Scale Scenarios “ International Journal of Recent Trends in Engineering, Vol. 1, No. 1, May 2009.

[3]. Julian Hsu et al, "Performance of Mobile Ad hoc networking routing protocols in large scale scenarios", vol 1, pages 21-24, proceedings of MILCOM 2004 -2004 IEEE Military Communications Conference.

[4]. A.Boomarani Malany, V.R.Sarma Dhulipala, and RM.Chandrasekaran "Throughput and Delay Comparison of MANET Routing Protocols" Int. J. Open Problems Compt. Math., Vol. 2, No. 3, September 2009.

[5]. Xiang Zeng, Rajive Bagrodia and Mario Gerla "Glomosim: A library for parallel simulation of large scale Wireless Networks".

[6]. Hong Jiang, J. J. Garcia-Luna-Aceves, "Performance Comparison of Three Routing Protocols for Ad Hoc Networks".

[7]. Lokesh Bajaj, Mineo Takai, Rajat Ahuja, Ken Tang, Rajive Bagrodia, and Mario Gerla "GloMoSim: A Scalable Network Simulation Environment".

[8]. R.Sivaraman, V.R.Sarma Dhulipala, L.Sowbhagya, B.Vishnu Prabha " Comparative Analysis of Qos Metrics in Mobile Ad Hoc Network Environment" International Journal of Recent Trends in Engineering, Vol 2, No. 4, November 2009.

[9]. Rajendra V. Boppana Anket Mathur, "Analysis of the Dynamic Source Routing Protocol for Ad Hoc Networks"

[10]. Stuart Kurkowski, Tracy Camp, Michael Cologrosso, "MANET Simulation Studies: The Incredible", Mobile Computing and Communications Review, Volume 9, Number 4, ACM, 2005.

[11]. Mohd Izuan Mohd Saad, "Performance Analysis of Random-Based Mobility Models in MANET Routing Protocol” European Journal of Scientific Research. 